\title{
Corticocortical feedback increases the spatial extent of normalization
}

\author{
Jonathan J. Nassi ${ }^{1+\neq}$, Camille Gómez-Laberge ${ }^{1,2 \neq}$, Gabriel Kreiman ${ }^{2,3}$ and Richard T. Born ${ }^{1 *}$ \\ ' Department of Neurobiology, Harvard Medical School, Boston, MA, USA \\ 2 Department of Ophthalmology, Boston Children's Hospital, Boston, MA, USA \\ ${ }^{3}$ Swartz Center for Theoretical Neuroscience, Harvard University, Cambridge, MA, USA
}

\section{Edited by:}

Lionel G. Nowak, Université

Toulouse - CNRS, France

Reviewed by:

Frédéric Chavane, Centre National de la Recherche Scientifique, France Guy A. Orban, University of Parma

Medical School, Italy

\section{*Correspondence:}

Richard T. Born, Department of

Neurobiology, Harvard Medical

School, 220 Longwood Ave.,

Boston, MA 02115, USA

e-mail: richard_born@

hms.harvard.edu

${ }^{\dagger}$ Present address:

Jonathan J. Nassi, Salk Institute for Biological Studies, San Diego, USA

¥ These authors have contributed equally to this work.
Normalization has been proposed as a canonical computation operating across different brain regions, sensory modalities, and species. It provides a good phenomenological description of non-linear response properties in primary visual cortex (V1), including the contrast response function and surround suppression. Despite its widespread application throughout the visual system, the underlying neural mechanisms remain largely unknown. We recently observed that corticocortical feedback contributes to surround suppression in $\mathrm{V} 1$, raising the possibility that feedback acts through normalization. To test this idea, we characterized area summation and contrast response properties in V1 with and without feedback from V2 and V3 in alert macaques and applied a standard normalization model to the data. Area summation properties were well explained by a form of divisive normalization, which computes the ratio between a neuron's driving input and the spatially integrated activity of a "normalization pool." Feedback inactivation reduced surround suppression by shrinking the spatial extent of the normalization pool. This effect was independent of the gain modulation thought to mediate the influence of contrast on area summation, which remained intact during feedback inactivation. Contrast sensitivity within the receptive field center was also unaffected by feedback inactivation, providing further evidence that feedback participates in normalization independent of the circuit mechanisms involved in modulating contrast gain and saturation. These results suggest that corticocortical feedback contributes to surround suppression by increasing the visuotopic extent of normalization and, via this mechanism, feedback can play a critical role in contextual information processing.

Keywords: visual cortex, corticocortical feedback, alert macaque, area summation, normalization

\section{INTRODUCTION}

Normalization has been advanced as a canonical computation in which a neuron's driving input is divided by the summed activity of a pool of neurons. This computational framework has been successfully applied throughout the visual system, across sensory modalities and in several different species (Carandini and Heeger, 2012). Response normalization has several attractive properties, particularly in sensory processing where it allows neurons with limited dynamic range to shift their response functions according to local statistics and reduces redundancy in neural population codes thus making them more efficient (Carandini et al., 1997).

Normalization has also been successful in explaining area summation properties in V1 (Cavanaugh et al., 2002), which are thought to contribute to a large spectrum of contextual effects in visual perception (Albright and Stoner, 2002). V1 neurons sum responses within the central region of their receptive field but display response suppression as stimuli invade the receptive field surround (Sceniak et al., 1999). Normalization can account for these non-linear responses, including the observation that reducing stimulus contrast increases the size of the summation field (Levitt and Lund, 1997). These effects of contrast on area summation properties are best captured by a model in which input drive and the normalization pool are stable in spatial extent but their relative gains depend on contrast (Cavanaugh et al., 2002).

Despite advancing our understanding of the phenomenological computations that subserve area summation, the actual neural mechanisms underlying normalization remain poorly understood. Circuits implementing normalization-like operations in V1 could be based on feed-forward signals combined with local non-linearities (Kayser et al., 2001), horizontal connections (Reynaud et al., 2012), or feedback signals from higher visual areas (Angelucci and Bressloff, 2006). While there is evidence that horizontal signals and, in particular, certain subtypes of interneurons are important in area summation properties (Adesnik et al., 2012), the extent to which feedback signals play a role is unclear. We recently showed that feedback from V2 and V3 contributes to surround suppression in V1 (Nassi et al., 2013): inactivation of feedback led to increased response magnitudes for large stimuli engaging the receptive field surround. The effect of feedback inactivation on surround modulation suggests that feedback may participate in normalization computations. 
This study begins with the premise that area summation arises at least in part due to a divisive normalization mechanism and does not attempt to rule out alternative models. We sought to characterize how well a normalization model could account for feedback's influence on surround suppression and how different components of the normalization operation were affected by feedback and contrast. To do so, we applied a standard normalization framework to area summation data (reported by Nassi et al., 2013) and to new contrast response data obtained in V1 of the alert macaque with feedback from V2 and V3 intact or inactivated by cooling. We found that the observed decrease in surround suppression strength during feedback inactivation was best captured by a reduction in the spatial extent of a divisive normalization pool. On the other hand, the contrast sensitivity of area summation in the same neurons was better accounted for by changes in gain. We confirmed this parametric dissociation by showing empirically that (1) the summation field expansion caused by contrast reduction and (2) the contrast response function within the summation field both remain intact without V2/V3 feedback. These results support the idea that corticocortical feedback contributes to surround suppression by increasing the spatial extent of normalization independent of bottom-up drive and, thus, identify feedback as a key circuit element of a canonical computation operating throughout the brain.

\section{MATERIALS AND METHODS}

All experiments were in accordance with the National Institutes of Health Guide for Care and Use of Laboratory Animals and were approved by the Harvard Medical Area Standing Committee on Animals.

\section{EXPERIMENTAL PREPARATION AND PROTOCOL}

The experimental preparation and associated protocols have been described previously in Nassi et al. (2013). The details relevant to the current study are described here. Two male macaque monkeys (Macaca mulatta; M and R) were implanted with a head post and a scleral search coil for monitoring eye position. They were trained to fixate within a $1-1.5^{\circ}$ window for $2-3 \mathrm{~s}$ for juice reward while seated in custom chairs (Crist Instrument). In each animal, three cryoloops were implanted in the lunate sulcus of the right hemisphere (Lomber et al., 1999) for reversible inactivation of V2 and V3 by cortical cooling, and a recording chamber (Crist Instrument) was implanted for electrophysiological access to V1 using tungsten microelectrodes (FHC). The extracellular recordings were acquired using a Cambridge Electronic Design 1401 system, and single- vs. multi-unit activity was determined using action potential waveform analysis software (Spike2).

Once a single-unit or multiunit cluster was isolated, we mapped the approximate borders of the receptive field [minimum response field (RF)], determined the optimal spatial and temporal frequencies, and preferred orientation/direction using a small grating. RFs were located at eccentricities between 2 and $6^{\circ}$, which were targeted so as to match the approximate retinotopy of regions in the lunate sulcus (V2/V3) in close proximity to the cryoloops. All subsequent tuning data were obtained using drifting sinusoidal gratings of mean luminance matching the surround $\left(42 \mathrm{~cd} / \mathrm{m}^{2}\right)$ at the optimal direction and spatiotemporal frequencies, centered in the RF, with a $0.04^{\circ}$ graded-contrast perimeter to reduce edge effects. Each stimulus presentation had a motiononset delay of $250 \mathrm{~ms}$ (monkey M) or $300 \mathrm{~ms}$ (monkey R) after appearance and then moved for either $750 \mathrm{~ms}$ (monkey $\mathrm{M}$ ) or $500 \mathrm{~ms}$ (monkey R). Tuning curves for the electrophysiology data were calculated from spike counts collected in the first $500 \mathrm{~ms}$ after motion onset. Between successive stimuli, we interposed $500 \mathrm{~ms}$ blanks at the mean luminance. Each stimulus condition was presented at least five times. The values of the parameter being varied in each experiment were always presented in block-wise random order.

Contrast response function data were obtained with sinusoidal gratings ranging in contrast from 0 to $99 \%$ in logarithmic steps. Area summation data were then obtained with sinusoidal gratings ranging in diameter from 0.125 to $8^{\circ}$ (monkey $\mathrm{M}$ ) or from 0.16 to $6.3^{\circ}$ (monkey $\mathrm{R}$ ) of visual angle in logarithmic steps. The full range of diameters was each presented at two different contrast levels, either in "block" design (monkey M) or randomly interleaved (monkey R). Differences in pre-stimulus firing rate (300-50 ms before stimulus onset) between contrast levels were not detected in either animal (Mann-Whitney $U$-test, each animal $p>0.1$ ). The two contrast levels were chosen to span the linear portion of the contrast response profile (i.e., just above the spontaneous firing rate and just below $90 \%$ of the maximum firing rate).

This protocol was repeated while chilled methanol was pumped through the cryoloops, effectively ceasing all visually evoked activity in the visual field representation of V2 and V3 that corresponded to the V1 recording site. Pre-cooling (Control) and cooling (Feedback inactive) recording sessions ran for 40-60 min in total. Post-cooling area summation measurements were possible in 19 units, which confirmed the reversibility of reported effects. A series of control studies were performed in our previous study to rule out the possibility of direct cooling effects in V1 (Nassi et al., 2013). Critically, we observed no effect of cooling in the upper bank of the calcarine sulcus in V1, where neurons have receptive fields outside of the feedback "scotoma." Furthermore, we could find no relationship between the magnitude of cooling effects and the proximity of recorded neurons to the V1/V2 border (details on experimental protocol and measurements of cortical temperature are found in Nassi et al., 2013).

\section{AREA SUMMATION MODEL}

We analyzed the area summation data from each unit using a ratio of Gaussians model (ROG; Cavanaugh et al., 2002) whose response $R_{\mathrm{ROG}}(x)$ is dependent on the stimulus diameter $x \geq 0$. This model implements the canonical normalization computation described by Carandini and Heeger (2012), which can be written as:

$$
R_{\mathrm{ROG}}(x)=R_{0}+\frac{D(x)}{\sigma+N(x)},
$$

where $R_{0}$ is the spontaneous rate, and $\sigma>0$ is the constant background normalization pool activity. The model consists of two area summation components: $D(x) \geq 0$ is the total excitatory drive, and $N(x) \geq 0$ is the normalization pool input. Both $D$ and $N$ respond to a stimulus of diameter $x \geq 0$ in proportion to its 
area under a Gaussian sensitivity function defined on the visual field. This form can be written in terms of the error function as

$$
\begin{aligned}
f(x) & =\frac{1}{\sqrt{\pi}} \int_{-x / 2}^{+x / 2} e^{-\left(\frac{y}{w}\right)^{2}} d y \\
& =w \cdot \operatorname{erf}(x / 2 w)
\end{aligned}
$$

where the spatial extent for $D$ and $N$ are individually determined by the parameters $w_{D}>0$ and $w_{N}>0$, respectively. A stimulus of size $x=w$ would lead to $52 \%$ of the area under this sensitivity function, making numerical estimates of $w_{D}$ and $w_{N}$ a practical benchmark of the drive and surround effective diameters. The underlying supposition is that summation relies heavily on the number of inputs from neurons with overlapping receptive fields: a large- $w$ neuron receives more input than a small- $w$ neuron and, thus, responds more vigorously over a larger visual field. Cavanaugh et al. (2002) had prescribed based on their data that both $D(x)$ and $N(x)$ be proportional to the square of the functions above, so that the model be explicitly written with subscripted gain parameters $k$ and size parameters $w$ as

$$
R_{\mathrm{ROG}}(x)=R_{0}+\frac{k_{D}\left[w_{D} \operatorname{erf}\left(x / 2 w_{D}\right)\right]^{2}}{\sigma+k_{N}\left[w_{N} \operatorname{erf}\left(x / 2 w_{N}\right)\right]^{2}}
$$

We set the constant $\sigma=1$ and also found that it was necessary for stabilizing responses to small stimulus diameters (which make the normalization term in the denominator approach zero). To avoid local optima in equation (3), we fitted parameters in three nested stages. First, all parameters were constrained as $0 \leq k<K$ and $0<w<W$, where the upper limits $K$ and $W$ were initially close to zero and progressively increased until the model fit mean-square-error (MSE) decreased to within 5\% of the asymptotic MSE (obtained without upper limits). This favored solutions with smaller, interpretable, $k$ and $w$ values and avoided situations where parameters grew only to marginally improve MSE without any obvious changes in the fitted curve. Second, for each set of limits $K$ and $W$, a grid of initial parameter values was used to identify the region where optimizations converge to the same solution. Third, we ensured that optimization steps were discretized according to the model's sensitivity to parameter changes, so that optimization steps in any parametric direction had equal effect on objective function output (the trust-region-reflective algorithm in Matlab's lsqcurvefit function can accomplish this by estimating the objective function's Jacobian matrix). Optimization was computationally demanding, so this routine was programmed for parallel processing on a high performance computer cluster.

\section{AREA SUMMATION CURVE FEATURES}

We defined the summation field size as the smallest diameter of the stimulus eliciting at least $95 \%$ of the peak response rate. Summation asymptote size was defined as the largest stimulus diameter that elicited a response $5 \%$ greater than the rate asymptote (when observable with largest diameters used). The strength of surround suppression was quantified with an index dependent on the response peak and asymptote spike rates: $S S I=$ $1-R_{\text {asym }} / R_{\text {peak }}$, defined for units satisfying $0<R_{\text {asym }} \leq R_{\text {peak }}$. In the absence of surround suppression, $R_{\mathrm{asym}}=R_{\mathrm{peak}}$ and so
$S S I=0$. As surround suppression becomes absolute $\left(R_{\mathrm{asym}} \rightarrow 0\right.$; $R_{\text {peak }}>0$ ), and so $S S I \rightarrow 1$ (see Figure 1B).

\section{CONTRAST RESPONSE MODEL}

Contrast sensitivity was quantified using the Naka-Rushton hyperbolic ratio function (Naka and Rushton, 1966) of the Michelson contrast $0 \leq c \leq 1$ given by

$$
R(c)=R_{0}+R_{\max } \frac{c^{n}}{c^{n}+\left(c_{50}\right)^{n}}
$$

where $R_{0}$ and $R_{\max }$ are the measured spontaneous and maximum response rates, respectively, and $n$ and $c_{50}$ are the model parameters quantifying contrast sensitivity and contrast-at-halfresponse, respectively. This optimization problem was convex and was solved by least-squares minimization.

\section{GOODNESS-OF-FIT INDICES}

These experiments required that the number of repeated trials for each data point be minimized $(n=5)$ in consideration of the large space of experimental conditions (i.e., grating size $\times$ contrast per baseline, cooling, and recovery block). Consequently, non-parametric methods like cross-validation were unattractive, since subsampled trials became too few to effectively average out across-trial fluctuations. We therefore opted to compare model configurations with equal number of parameters based on their percentage of variance explained. With an unequal number of parameters, we used a $\chi_{N}^{2}$ statistic comparing model and measured rates, normalized by the optimization problem's degrees of freedom: $d f=N_{\text {stimuli }}-N_{\text {parameters }}+1$, where 1 is for the measured spontaneous rate (Hoel et al., 1971). Such parametric methods continue to be used in similar studies probing the effects of multiple experimental conditions on area summation (Carandini et al., 1997; Cavanaugh et al., 2002, 2012; Roberts et al., 2007; Olsen et al., 2010; Nienborg et al., 2013; Vaiceliunaite et al., 2013).

\section{STATISTICS}

Values-in-text are reported as mean $\pm S D$ unless otherwise indicated. Goodness-of-fit comparisons and differential-responses to stimulus contrast, size, and feedback conditions were evaluated using ANOVA. Across-condition differences in summation features or spatial parameters $(w)$ were evaluated with a two-tailed $t$-test. The gain parameters $(k)$ were decidedly non-normal (spanning $\sim 2$ orders of magnitude) and, thus, were evaluated with the Mann-Whitney non-parametric $U$-test. The significance of Pearson correlation coefficient estimates $r$ was determined following a $t$ statistic conversion with $\mathrm{H}_{0}: \rho=0$. Effects were considered significant for $p<0.05$, and multiple simple-effect comparisons were accounted by Tukey's HSD test.

\section{RESULTS}

\section{EFFECTS OF FEEDBACK INACTIVATION ON AREA SUMMATION}

We investigated how the area summation properties observed in $\mathrm{V} 1$ depend on corticocortical feedback from V2 and V3. To do so, we reversibly inactivated parts of V2 and V3 using "cryoloops" chronically implanted in the right lunate sulcus of two alert, fixating monkeys as reported in Nassi et al. (2013) (monkeys M 
A

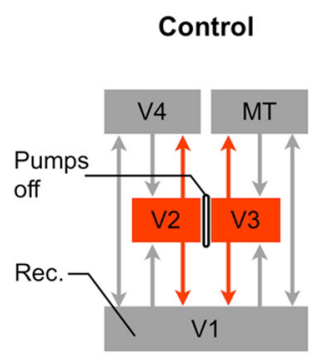

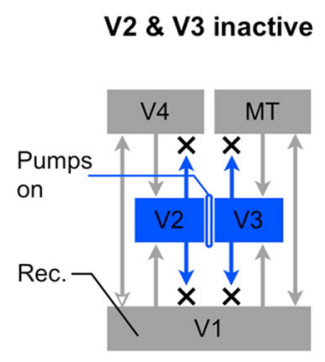

C

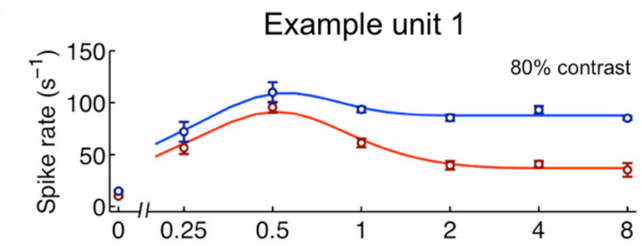

B

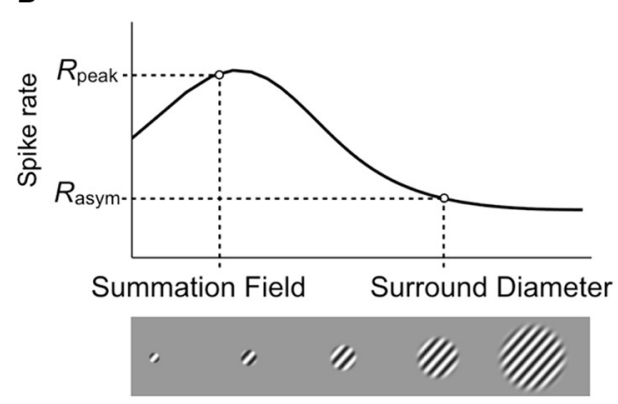

Example unit 2

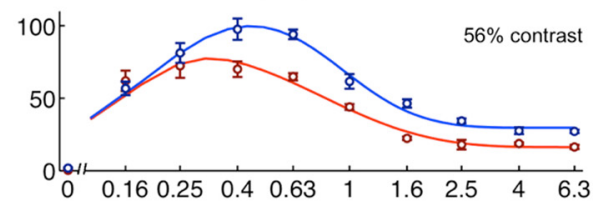

$10 \%$ contrast

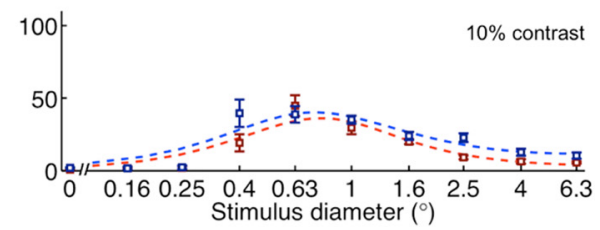

FIGURE 1 | Cortical feedback influences area summation in V1.

(A) Feedback to $\mathrm{V} 1$ was reversibly inactivated by cooling $\mathrm{V} 2$ and $\mathrm{V} 3$ using cryoloops (Materials and Methods). (B) Schematic of area summation curve measured using drifting gratings of various sizes, centered on the receptive field. The summation field, surround diameter, and their corresponding spike rates $\left(R_{\text {peak }}, R_{\text {asym }}\right)$ are the main features characterizing the curve's shape. (C) Area summation curves from two example units during control (red) and feedback inactive (blue) conditions. Data are shown as mean \pm s.e.m. $(n=5)$. The curves represent the fit from the model in Figure 2A. (D) Area summation curves measured from same units using low contrast gratings. The typical effect of feedback inactivation was an increase in response magnitude, particularly for high contrast gratings whose diameter extended beyond the summation field. The increased rate effect was present, yet diminished, during low contrast visual stimulation. and R; Figure 1A). Pumping chilled methanol through these loops cooled surrounding cortex down to temperatures below $20^{\circ} \mathrm{C}$ and allowed us to temporarily eliminate visually evoked activity across visuotopic regions of V2 and V3 overlapping and extending beyond the receptive field locations of recorded units in V1 (see Materials and Methods).

Area summation curves from 64 units (36 single units, 28 multi-units) in V1 were measured before and during inactivation of feedback by presenting drifting sinusoidal gratings centered within the receptive field and varying in size. The gratings were set to the preferred direction and spatiotemporal frequency for each unit. Due to the known effects of contrast on area summation (Sceniak et al., 1999), we tested effects of feedback inactivation at two contrast levels ("high" and "low;" see Materials and Methods). In this section, we analyze feedback inactivation effects at both contrast levels independently. In the subsequent section, we determine whether and how feedback inactivation interacts with the effects of contrast on area summation. All 64 units were sensitive to the size of the stimulus (Two-Way ANOVA, main effect stimulus diameter, each $p<0.05)$. In all cases the neural response initially increased as stimulus size increased from the smallest size tested (illustrated schematically in Figure 1B), peaked for stimuli of intermediate size and were almost always reduced for larger sizes. The observed response profile-characterized by initial summation followed by surround suppression-is typical for neurons in V1 (Sceniak et al., 1999). Feedback inactivation altered the overall response magnitude in 40 of 64 units (main effect feedback, each $p<0.05$ ), increasing responses on average, and interacted with stimulus diameter in 29 of 64 units (diameter $\times$ feedback interaction, each $p<0.05$ ). As reported previously, the most common effect of inactivation was an increase in response magnitude, particularly for grating diameters that extended beyond the borders of the receptive field center (Figure 1C) (Nassi et al., 2013).

In order to investigate the underlying mechanisms that give rise to the observed effects of feedback inactivation, we fit the area summation data with the "ratio of Gaussians" (ROG) model (Cavanaugh et al., 2002), a form of divisive normalization that implements an excitatory drive component $D(x) \geq 0$, dependent on the stimulus diameter $x$, whose output is divided by the diameter-dependent activity $N(x) \geq 0$ and background activity $\sigma>0$ of the unit's normalization pool (Figure 2A). Under the assumptions of this model, it is possible to infer the response gain and visual field extent of the excitatory drive $\left\{k_{D}, w_{D}\right\}$ and normalization pool $\left\{k_{N}, w_{N}\right\}$ components accounting for receptive field center-surround interactions. We fit the model to the responses of each unit independently by allowing all parameters to vary for both feedback conditions. This unbiased 

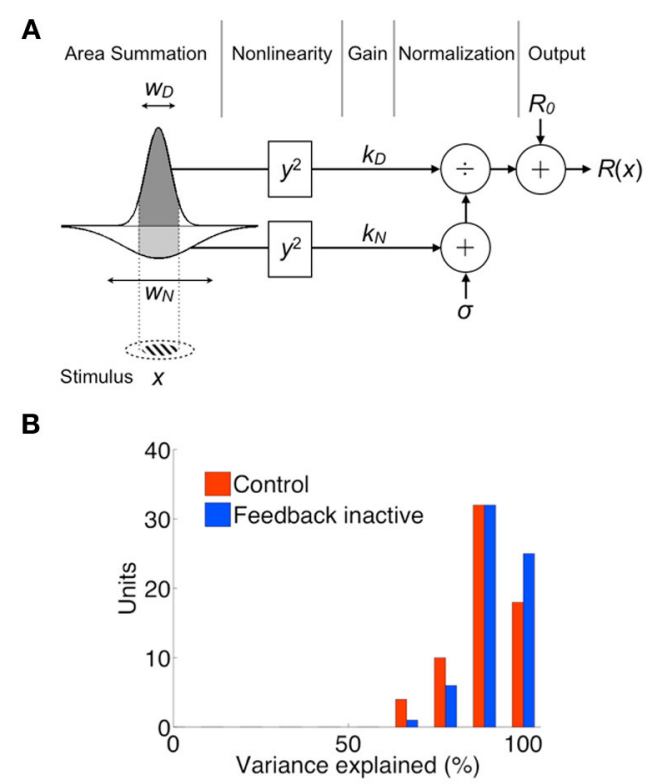

FIGURE 2 | Normalization area summation model (ratio of Gaussians). (A) Schematic representation of the model. The receptive-field-centered grating stimulus of diameter $x$ determines the integration bounds of the excitatory drive (dark shading) and normalization pool (light shading) area summation components, characterized by spatial parameters $w_{D}$ and $w_{N}$ respectively. Their outputs are squared and amplified by gains $k_{D}$ and $k_{N}$ before normalization. The constant $\sigma$ represents baseline normalization pool activity and is set to unity without loss of generality. $R_{0}$ is the

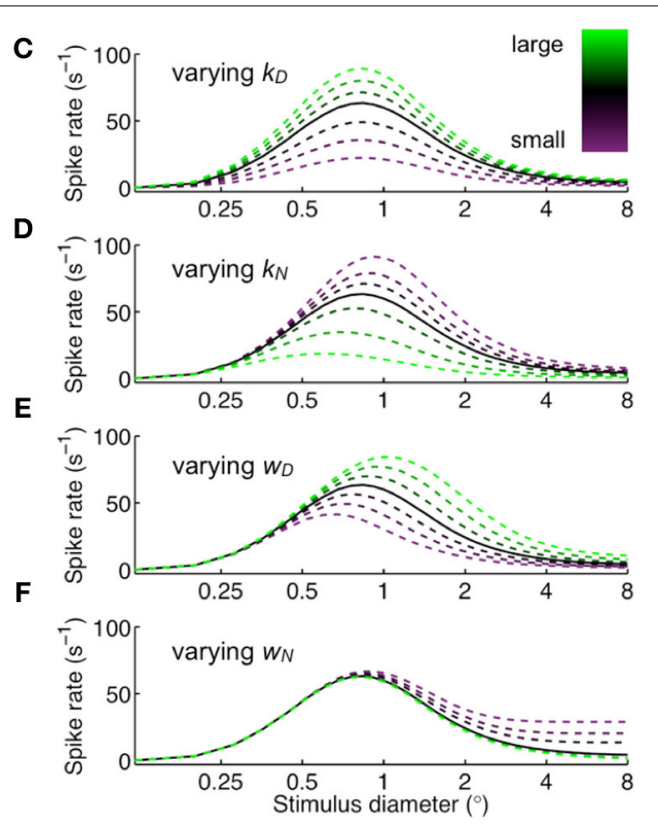

measured spontaneous rate, and $R(x)$ is the response spike rate. (B) Summary of model goodness-of-fit per unit for both control (red) and feedback inactive (blue) conditions (median variance explained: $90 \%$ control, 93\% feedback inactive). (C-F), Illustration of area summation curve effects due to individual variation of each model parameter.

Population-averaged parameters produce solid black curve, and the effects of increasing/decreasing one parameter are plotted as green/purple broken curves. approach is best when probing for a link between an experimental effect and a parameter, provided that measures are taken to avoid redundancy/trade-offs between parameters as well as to counter-balance potential differences in parameter sensitivity (see Materials and Methods). Potential links between experimental conditions and parameters were further tested by comparing constrained models. Although the number of parameters was large relative to the data points (8 parameters for $12-18$ data points), each parameter's influence on the area summation curves was well constrained-only $k_{N}$ and $w_{D}^{-1}$ had qualitatively similar influences, yet even in this case $k_{N}$ primarily affects peak rate and $w_{D}$ primarily summation field size (Figures 2D,E). This version of the normalization model was shown to be optimal in accounting for similar data in anesthetized macaques (Cavanaugh et al., 2002). The ROG model accounted well for the area summation data with and without feedback, explaining $90 \%$ of the variance overall across the population both in the feedback and control conditions (Figure 2B, see example fits in Figures 1C,D).

From the fitted area summation curves, we measured several features (illustrated in Figure 1B): the summation field diameter (SF), the surround diameter and the respective peak and asymptotic response rates $\left(R_{\text {peak }}, R_{\text {asym }}\right)$. Each of these features was compared across the population for control and feedback-inactivated conditions (Table 1). For high contrast stimuli (Figures 3A-D), the most prominent effect of feedback inactivation was an increase in $R_{\text {asym }}$ of 7 spikes/s on average (67\% increase from control; $p<0.001$; Figure 3C). This caused the surround suppression index (SSI) to fall on average by $8 \%(p<0.001$; Figure 3D). For low contrast stimuli, asymptote rates increased as well; however, a concomitant increase in peak response rates resulted in only a $3 \%$ reduction in SSI $(p=0.10$; Table 1). We also observed additional effects during high contrast stimulation: an increase in $\mathrm{SF}$ of $0.04^{\circ}$ on average $(p=0.01$; Figure 3A) and an average decrease in surround diameter of $0.3^{\circ}$ ( $p=0.009$; Table 1). These latter changes, however, were small in comparison to the reduction in surround suppression observed for high contrast stimuli. Together, these results indicate that the main effect of feedback inactivation on area summation in V1 is to increase responses to large stimuli and therefore reduce the overall strength of surround suppression (Nassi et al., 2013).

To gain insight into the mechanisms underlying the effects of feedback inactivation, we examined how the variation of each model parameter influences the area summation curve (Figures 2C-F). These figures show that the normalization pool's spatial extent $w_{N}$ alone could account for the effect of feedback inactivation, since reducing $w_{N}$ leads to an increased response for large stimuli extending into the receptive field surround (i.e., increased $R_{\text {asym }}$ ), while leaving the responses to small stimuli confined to the center unchanged (Figure 2F). By contrast, changes in the gain parameters $\left(k_{D}, k_{N}\right)$ and the excitatory drive's spatial extent $\left(w_{D}\right)$ are unable to affect responses to large stimuli without also affecting responses to smaller stimuli (Figures 2C-E). Not only do the other parameters primarily affect $R_{\text {peak }}$, but $k_{N}$ and $w_{D}$ also tend to produce sizeable shifts in SF, both of which 
were not typically observed in our data. When we compared the parameter estimates from the model across feedback conditions at high contrast, $w_{N}$ was significantly reduced during feedback inactivation from $1.96 \pm 1.29^{\circ}$ to $1.72 \pm 1.22^{\circ}$ (mean $\pm S D$,

Table 1 | Feedback's effect on area summation curve features and model parameters.

\begin{tabular}{lccc}
\hline High contrast & Control & Feedback inactive & p-value \\
\hline Summation field diameter $\left(^{\circ}\right)$ & $0.46 \pm 0.20$ & $0.51 \pm 0.22$ & 0.01 \\
Peak response rate $\left(\mathrm{s}^{-1}\right)$ & $54 \pm 32$ & $59 \pm 35$ & 0.06 \\
Surround diameter $\left(^{\circ}\right)$ & $4.4 \pm 1.5$ & $4.1 \pm 1.6$ & 0.009 \\
Asymptote rate $\left(\mathrm{s}^{-1}\right)$ & $12 \pm 15$ & $19 \pm 20$ & $<0.001$ \\
SSI (a.u.) & $0.79 \pm 0.17$ & $0.71 \pm 0.22$ & $<0.001$ \\
$k_{D}($ a.u. & $1730[3110]$ & $1770[2440]$ & 0.7 \\
$k_{N}($ a.u. & $12.9[13.8]$ & $7.99[14.5]$ & 0.2 \\
$W_{D}\left(^{\circ}\right)$ & $0.32 \pm 0.25$ & $0.31 \pm 0.18$ & 0.8 \\
$W_{N}\left({ }^{\circ}\right)$ & $1.96 \pm 1.29$ & $1.72 \pm 1.22$ & 0.006 \\
\hline Low contrast & Control & Feedback inactive & $\boldsymbol{p}$-value \\
\hline Summation field diameter $\left({ }^{\circ}\right)$ & $0.71 \pm 0.65$ & $0.72 \pm 0.38$ & 0.9 \\
Peak response rate $\left(\mathrm{s}^{-1}\right)$ & $32 \pm 22$ & $37 \pm 27$ & 0.01 \\
Surround diameter $\left({ }^{\circ}\right)$ & $4.6 \pm 1.6$ & $4.7 \pm 1.5$ & 0.6 \\
Asymptote rate $\left(\mathrm{s}^{-1}\right)$ & $9.6 \pm 12$ & $12 \pm 14$ & 0.01 \\
SSI (a.u.) & $0.71 \pm 0.26$ & $0.68 \pm 0.25$ & 0.10 \\
$k_{D}($ a.u. & $532[613]$ & $450[817]$ & 1 \\
$k_{N}($ a.u. & $5.02[5.89]$ & $3.79[5.82]$ & 0.1 \\
$W_{D}\left({ }^{\circ}\right)$ & $0.39 \pm 0.29$ & $0.40 \pm 0.20$ & 0.8 \\
$W_{N}\left({ }^{\circ}\right)$ & $2.14 \pm 1.31$ & $2.11 \pm 1.33$ & 0.6 \\
\hline
\end{tabular}

Mean $\pm S D$ and t-test used everywhere except for $k_{D}$ and $k_{N}$ reported as median [IQR] and Mann-Whitney U-test. $p=0.006$; Figure $3 \mathbf{H})$. The reduction in $w_{N}$ was not observed for low contrast stimulation $\left(2.14 \pm 1.31^{\circ}\right.$ vs. $2.11 \pm 1.33^{\circ}, p=0.6$; Table 1). This is not surprising given the much weaker effects of feedback inactivation at low contrast. Nevertheless, we did detect a population-wide relationship between feedback-induced changes in $w_{N}$ and SSI for both high and low contrast conditions $\left(r_{\text {high }}=0.30, r_{\text {low }}=0.29\right.$, each $\left.p<0.05\right)$. In agreement with Figures $2 \mathbf{C}-\mathbf{F}$, the remaining parameters $k_{D}, k_{N}$, and $w_{D}$ showed no trend of systematic change during feedback inactivation at either of the two contrast levels tested (Mann-Whitney $U$-test for $k$, and $t$-test for $w$ parameters, each $p>0.1$; Figures 3E-G).

According to the area summation model results (Figure 3 ), the effects of feedback inactivation on area summation were best captured by changes in the normalization pool's spatial extent $\left(w_{N}\right)$. In order to further determine the individual contributions of each parameter, we compared versions of the model in which only one of the four parameters was fixed across feedback conditions and the other three parameters could freely vary. Only small reductions in percentage of variance explained were observed when fixing any single parameter, primarily because all models captured the main curve features well. Nevertheless, we detected significant differences among these four fixed-parameter models (One-Way ANOVA, main effect of model, $p=0.001$; Table 2). Fixing $w_{N}$ resulted in the worst fits to the data (Tukey's test on fixed- $w_{N}$ simple effects, all $p<0.05$ ), while differences in fit quality were not detected for the three remaining fixed-parameter models (main effect excluding fixed- $w_{N}$ model, $p=0.8$ ). The differences among the fixed-parameter models underscore that changes in $w_{N}$ were important to capture the effects of feedback inactivation. In the context of the normalization model, our results indicate that the effects of feedback inactivation on area summation are best
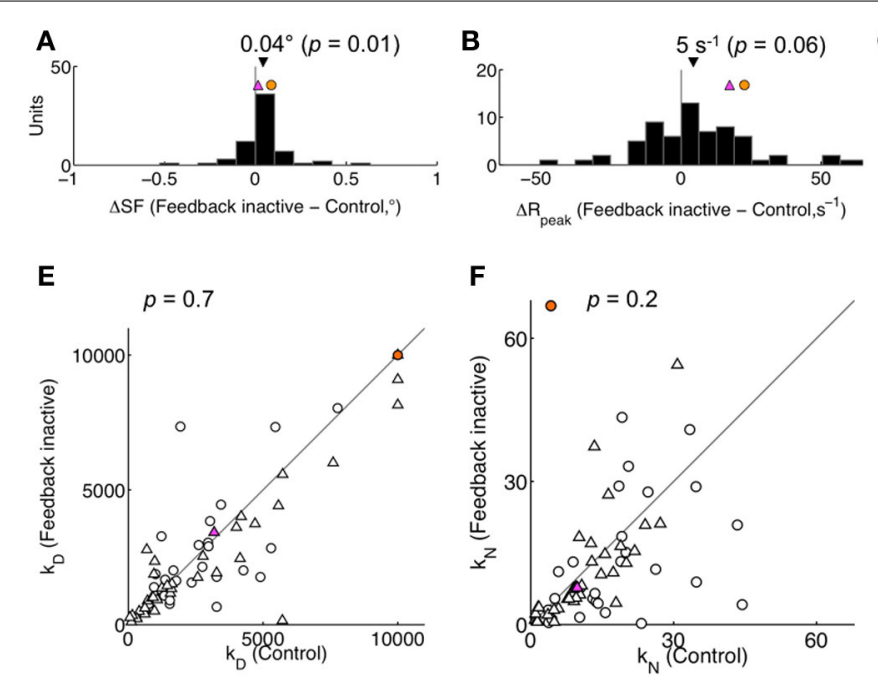

FIGURE 3 | Population effect of feedback inactivation on high contrast area summation. (A-D) Summation curve features: difference in summation field (SF), peak response rate $\left(R_{\text {peak }}\right)$, asymptote response rate $\left(R_{\text {asym }}\right)$, and surround suppression index (SSI) between feedback inactive and control conditions. Black triangle indicates population mean (with two-tailed $t$-test $p$-value). (E-H) Parameter estimates $\left(k_{D}, k_{N}, w_{D}, w_{N}\right)$ for all units plotted for Feedback inactive vs. Control conditions. $p$-values correspond to a two-tailed
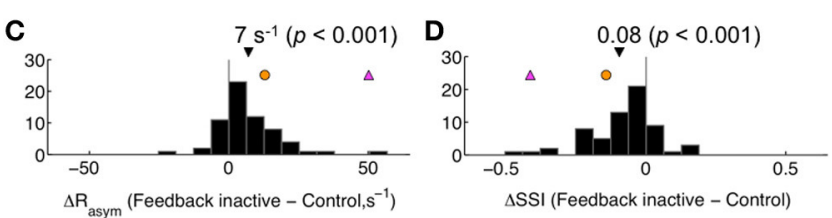

G
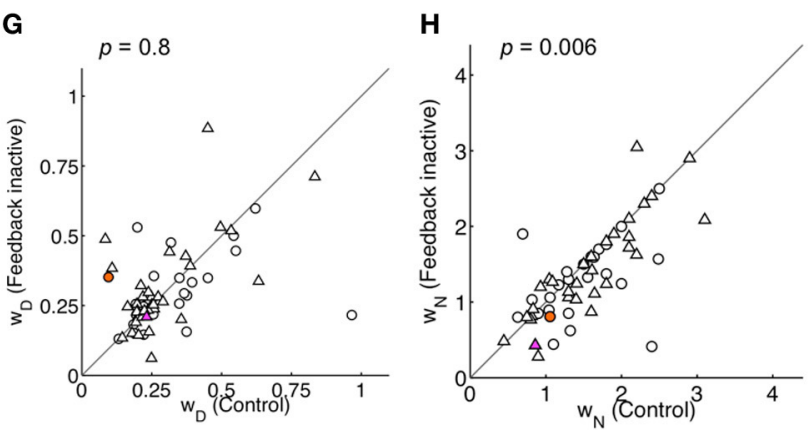

Mann-Whitney $U$-test for $k_{D}$ and $k_{N}$, and a $t$-test for $w_{D}$ and $w_{N}$. Triangles and circles indicate single- and multi-units, respectively. Differences were not detected in any feature (A-D) or parameter (E-H) when comparing singleand multi-unit subpopulations (each $p>0.05$ ). In all panels, the magenta and orange symbols indicate example units 1 and 2 in Figure 1. Feedback inactivation reduced SSI by increasing $R_{\text {asym }}$. The model accounted for this effect by systematically reducing $w_{N}$ across the population. 
explained by a reduction in the spatial extent of the normalization pool.

\section{CONTRAST GAIN MECHANISM UNALTERED DURING FEEDBACK INACTIVATION}

The above analysis suggests that feedback inactivation had little or no effect on the gains of the underlying excitatory drive and normalization pool components. In order to test this possibility further, we analyzed the effects of stimulus contrast on area summation, both with and without V2/V3 feedback, which have previously been shown to be mediated by changes in gain (Cavanaugh et al., 2002). If feedback effects are indeed independent of these gain mechanisms, we would expect the effects of stimulus contrast to be unaltered during feedback inactivation. We found that contrast had a significant effect on the area summation data for the majority of the population both with feedback intact (55 of 64 units) and inactivated (47 of 64 units) (TwoWay ANOVA, diameter $\times$ contrast interaction, each $p<0.05)$. Consistent with previous reports, the typical effect observed when lowering contrast was an expansion of the summation field and a reduction in peak response rate (Figure 4) (Sceniak et al., 1999). These effects were observed independently of feedback state. We quantified these effects at the population level using the area summation curve features obtained from the area summation model (Table 3). During the control condition, we confirmed that reducing stimulus contrast significantly increased
SF from $0.46 \pm 0.20^{\circ}$ to $0.71 \pm 0.65^{\circ}(p=0.002$; Figure 5A) and significantly decreased $R_{\text {peak }}$ from $54 \pm 32 \mathrm{~s}^{-1}$ to $32 \pm 22$ $\mathrm{s}^{-1}(p<0.001$; Figure 5B). The magnitude of these contrastinduced shifts in summation field diameter and peak response rate did not differ between feedback conditions $(\triangle \mathrm{SF}:-0.25 \pm$ $0.60^{\circ}$ vs. $-0.21 \pm 0.28^{\circ}, \Delta R_{\text {peak }}: 22 \pm 20 \mathrm{~s}^{-1}$ vs. $22 \pm 21 \mathrm{~s}^{-1}$, feedback on vs. off respectively, each $p>0.1$; Figures 6A,B), suggesting that the effect of contrast on area summation remained largely intact in the absence of feedback. In addition, there was a smaller contrast-induced effect on $R_{\text {asym }}$ (Figure 5C). The contrast-induced decrease in $R_{\text {asym }}$ was larger during feedback inactivation than during the control condition. This asymmetry explains why reducing stimulus contrast significantly decreased SSI during the control condition (average $8 \%$ decrease, $p<0.001$; Figure 5D) but not during feedback inactivation (average 3\% decrease, $p=0.14$; Table 3 ).

To gain insight into potential mechanisms underlying the observed contrast-induced effects, we re-examined how the variation of each model parameter influences the area summation curve (Figures 2C-F). Changes in any single parameter cannot reproduce the summation field increase and peak response rate decrease observed when reducing contrast. However, both effects can be simultaneously achieved by changing pairs of parameters. One possibility is that stimulus contrast simply regulates both $k_{D}$ and $k_{N}$ : a gain-specific mechanism. Alternatively, lowering stimulus contrast might be accounted for by a decrease in

Table 2 | Model comparisons across feedback conditions.

\begin{tabular}{|c|c|c|c|c|c|}
\hline & Area summation & $k_{D}$ fixed & $k_{N}$ fixed & $w_{D}$ fixed & $w_{N}$ fixed \\
\hline One-Way ANOVA (main effect): & & & & & $p=0.001$ \\
\hline One-Way ANOVA (main effect without $w_{N}$ fixed): & & & & & $p=0.8$ \\
\hline
\end{tabular}

Variance explained by the area summation model and the four versions that fix one parameter across feedback conditions.

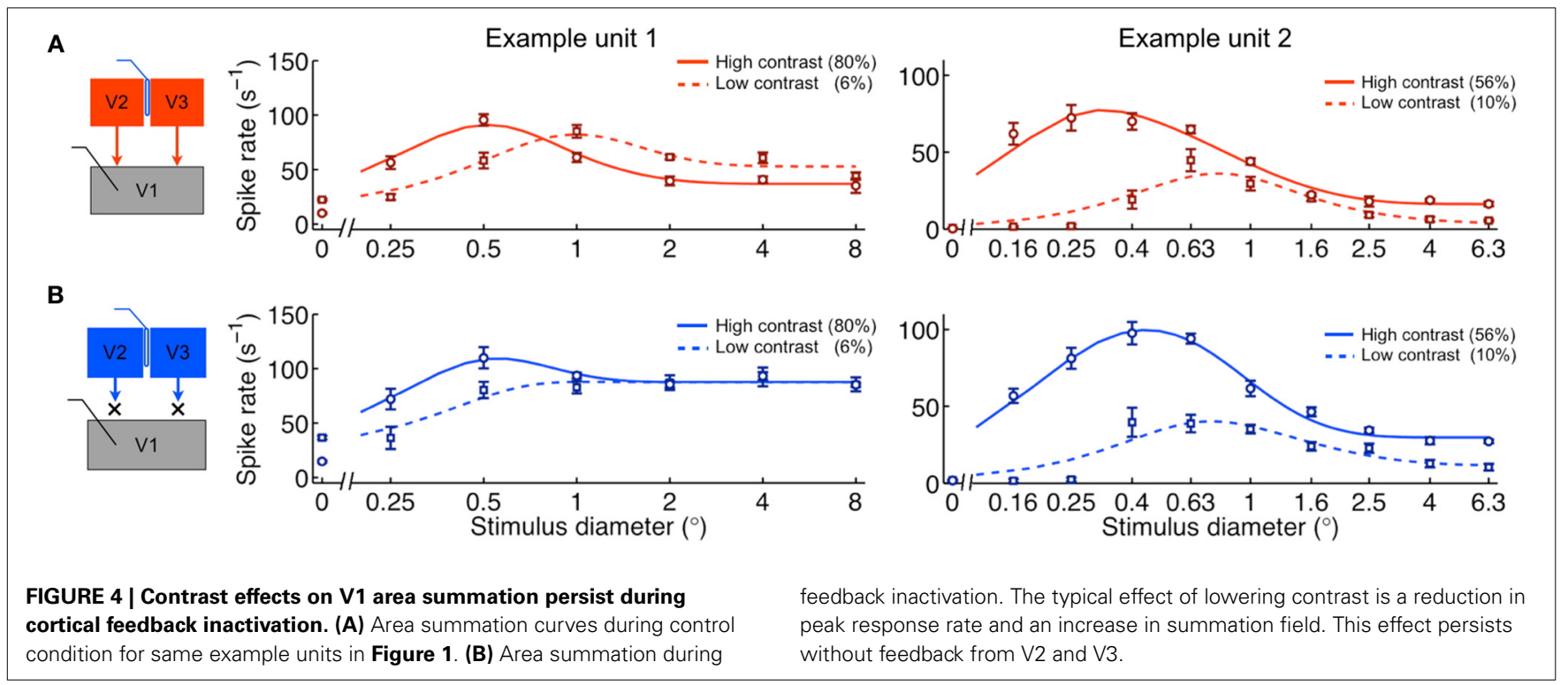


$k_{D}$ and an increase in $w_{D}$ : an input-drive-specific mechanism. To distinguish between these and other possibilities, we first compared changes in all parameters estimated for high vs. low contrast during the control condition (Table 3). Lowering contrast

Table 3 | Contrast's effect on area summation curve features and model parameters.

\begin{tabular}{|c|c|c|c|}
\hline Control condition & High contrast & Low contrast & $p$-value \\
\hline Peak size $\left({ }^{\circ}\right)$ & $0.46 \pm 0.20$ & $0.71 \pm 0.65$ & 0.002 \\
\hline Peak rate $\left(s^{-1}\right)$ & $54 \pm 32$ & $32 \pm 22$ & $<0.001$ \\
\hline Asymptote size $\left({ }^{\circ}\right)$ & $4.4 \pm 1.5$ & $4.6 \pm 1.6$ & 0.09 \\
\hline Asymptote rate $\left(\mathrm{s}^{-1}\right)$ & $12 \pm 15$ & $9.6 \pm 12$ & 0.006 \\
\hline SSI (a.u.) & $0.79 \pm 0.17$ & $0.71 \pm 0.26$ & $<0.001$ \\
\hline$k_{D}$ (a.u.) & $1730[3110]$ & 532 [613] & $<10^{-10}$ \\
\hline$k_{N}$ (a.u.) & 12.9 [13.8] & $5.02[5.89]$ & $<10^{-5}$ \\
\hline$w_{D}\left(^{\circ}\right)$ & $0.32 \pm 0.25$ & $0.39 \pm 0.29$ & $<0.001$ \\
\hline$w_{N}\left({ }^{\circ}\right)$ & $1.96 \pm 1.29$ & $2.14 \pm 1.31$ & 0.03 \\
\hline Feedback inactive & High contrast & Low contrast & $p$-value \\
\hline Peak size $\left({ }^{\circ}\right)$ & $0.51 \pm 0.22$ & $0.72 \pm 0.38$ & $<0.001$ \\
\hline Peak rate $\left(s^{-1}\right)$ & $59 \pm 35$ & $37 \pm 27$ & $<0.001$ \\
\hline Asymptote size $\left({ }^{\circ}\right)$ & $4.1 \pm 1.6$ & $4.7 \pm 1.5$ & 0.001 \\
\hline Asymptote rate $\left(\mathrm{s}^{-1}\right)$ & $19 \pm 20$ & $12 \pm 14$ & $<0.001$ \\
\hline SSI (a.u.) & $0.71 \pm 0.22$ & $0.68 \pm 0.25$ & 0.14 \\
\hline$k_{D}$ (a.u.) & 1770 [2440] & 450 [817] & $<10^{-8}$ \\
\hline$k_{N}($ a.u. $)$ & $7.99[14.5]$ & $3.79[5.82]$ & $<0.001$ \\
\hline$w_{D}\left({ }^{\circ}\right)$ & $0.31 \pm 0.18$ & $0.40 \pm 0.20$ & $<0.001$ \\
\hline$w_{N}\left({ }^{\circ}\right)$ & $1.72 \pm 1.22$ & $2.11 \pm 1.33$ & 0.003 \\
\hline
\end{tabular}

Mean $\pm S D$ and t-test used everywhere except for $k_{D}$ and $k_{N}$ reported as median [IQR] and Mann-Whitney U-test. caused both gain parameters to decrease (Figures 5E,F) and both size parameters to increase (Figures 5G,H). However, the effect was at least three times larger for the gain parameters than the field diameter parameters: $\Delta k_{D}=-73 \%, \Delta k_{N}=-62 \%$, $\Delta w_{D}=21 \%, \Delta w_{N}=9 \%$ ( $\Delta$ indicates change from high to low contrast), and the observed effects in gain parameters were more consistent across the population: $k_{D} \downarrow 95 \%$ units, $k_{N} \downarrow$ $83 \%$ units, $w_{D} \uparrow 23 \%$ units, $w_{N} \uparrow 38 \%$ units (arrow indicates direction of parameter change followed by percentage of units). The most likely interpretation is that stimulus contrast primarily

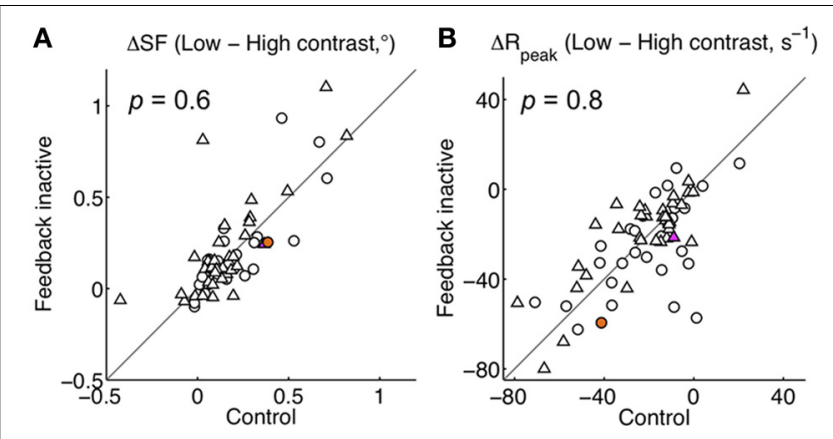

FIGURE 6 | Contrast-induced effects remain largely intact during feedback inactivation. (A) Shift in summation field from high to low contrast $(\Delta S F)$ plotted per unit for Feedback inactive vs. Control conditions. (B) Corresponding shift in the peak response rate $\left(\Delta R_{\text {peak }}\right)$. For both panels $p$-values correspond to a two-tailed $t$-test between feedback conditions. The magenta and orange symbols indicate shifts for the example units in Figure 1. No systematic change in contrast-induced effects were detected when feedback was inactivated.

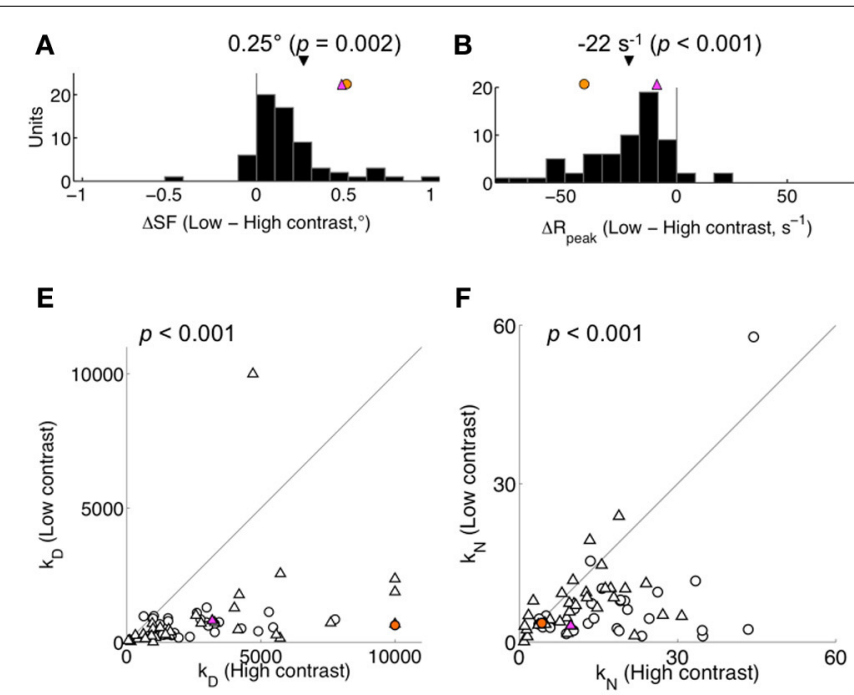

FIGURE 5 | Population effect of stimulus contrast on area summation during the control condition. (A-D) Summation curve features: difference in summation field (SF), peak response rate $\left(R_{\text {peak }}\right)$, asymptote response rate $\left(R_{\text {asym }}\right)$, and surround suppression index (SSI) between high and low contrast stimuli. Black triangle indicates population mean (with two-tailed $t$-test p-value). (E-H) Parameter estimates $\left(k_{D}, k_{N}, w_{D}, w_{N}\right)$ for all units plotted for high contrast vs. low contrast conditions. $p$-values correspond to a two-tailed Mann-Whitney $U$-test for $k_{D}$ and $k_{N}$, and a $t$-test for $w_{D}$ and $w_{N}$. Triangles
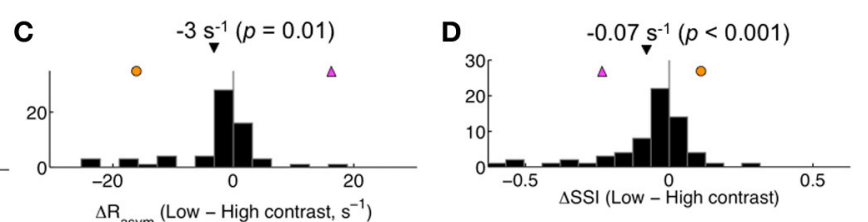

G
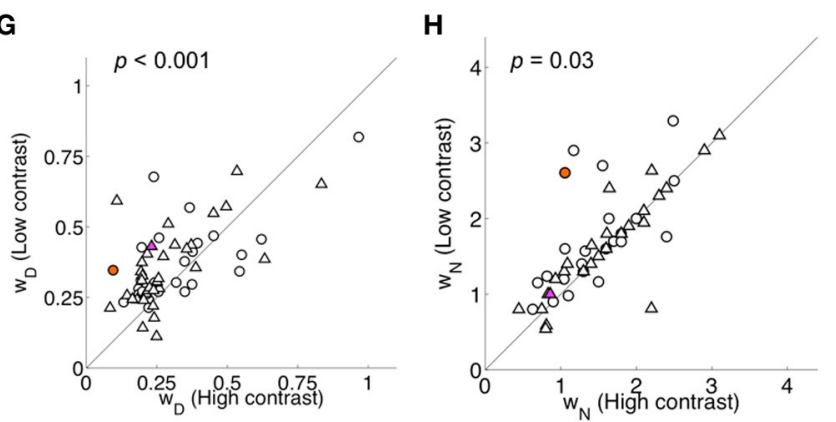

and circles indicate single- and multi-units, respectively. Differences were not detected in any feature (A-D) or parameter (E-H) when comparing across single- and multi-unit subpopulations (each $p>0.05$ ). In all panels, the magenta and orange symbols indicate features for example units in Figure 1. Reducing contrast increased the summation field, decreased the peak response rate, and yielded a small decrement in the asymptotic rate and suppression index. Both gain parameters increased and $w_{D}$ decreased with high contrast. 
engages a gain-specific mechanism of area summation, whereby both the excitatory drive and normalization pool signals scale in proportion to stimulus contrast.

To further evaluate the possibility of a contrast-related gain mechanism, we followed the approach of Cavanaugh et al. (2002) by comparing three fixed-parameter versions of the area summation model for each unit. The uniform model varies $k_{D}$ only; the gain model varies $\left\{k_{D}, k_{N}\right\}$, and the size model varies $\left\{k_{D}, k_{N}, w_{D}\right\}$. Each version of the model is rendered more complex than the previous one by allowing an additional parameter to vary across contrast conditions. Note that the sequence of adding parameters here follows the magnitude of the effects described in the previous paragraph when examining changes in individual parameters $\left(\Delta k_{D}, \Delta k_{N}, \Delta w_{D}, \Delta w_{N}\right)$. Therefore, our approach progressively makes the models more complex by allowing the next best-available parameter to vary across contrast. Model comparisons were again judged in terms of goodness-of-fit, but this time, in addition to percentage of variance explained, we used the $\chi_{N}^{2}$ statistic in order to account for the differing degrees of freedom across models (see Materials and Methods). In agreement with Cavanaugh et al. (2002), we found that the gain model was most efficient in explaining the control data in terms of variance explained (Figure 7A) and $\chi_{N}^{2}$, the goodness-of-fit per $d f$ (uniform $\chi_{N}^{2}=5.60$; gain $\chi_{N}^{2}=4.78$; size $\chi_{N}^{2}=5.13$; see Materials and Methods). Therefore, during control conditions, the effects of contrast on area summation were best explained by changes in the gains of the underlying excitatory drive and normalization components, with no need to invoke any changes in their sizes. During feedback inactivation, we again found that the gain model was the best at explaining the effect on contrast (uniform $\chi_{\mathrm{N}}^{2}=4.65$; gain $\chi_{\mathrm{N}}^{2}=3.79$; size $\chi_{N}^{2}=3.95$; Figure $7 \mathbf{B}$ ). Indeed, fixing $w_{D}$ and $w_{N}$ reduced the variance explained by only a few percent, and their feedbackinduced parameter shifts were qualitatively similar (Table 4). These results show that the effect of stimulus contrast on area
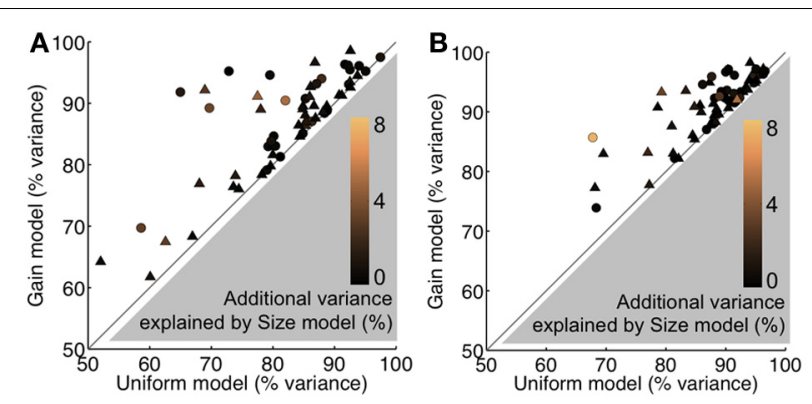

FIGURE 7 | Constrained model comparisons. (A) Variance explained per unit during the Control condition, plotted for the gain model vs. uniform model. Color scale indicates additional variance explained by the size model relative to gain model. Gray region cannot be occupied because the gain model has one additional parameter than the uniform model. Similarly, the color scale is non-negative because the size model has one additional parameter than the gain model. (B) Same format as (A) for the Feedback inactivation condition. The gain model considerably improves the goodness-of-fit, while only slight improvements are observed with the size model. For all panels, triangles and circles indicate single- and multi-units, respectively. summation in V1, as well as the underlying gain-related mechanism, remains largely intact in the absence of feedback from V2 and $\mathrm{V} 3$.

\section{CENTER CONTRAST SENSITIVITY UNALTERED DURING FEEDBACK INACTIVATION}

The above results show that feedback inactivation had little or no effect on the gains of the underlying excitatory drive and normalization pool components. If this is indeed the case, we would not expect to observe population-wide changes in contrast sensitivity for small stimuli restricted to the receptive field center. We measured the contrast response function for 36 units from the same two monkeys (24 single units, 12 multi-units). The experimental protocol was identical to that used for area summation measurements, except that gratings were fixed in size, appeared entirely within the experimentally-derived high contrast summation field, and eight contrast values were pseudo-randomly presented. All 36 units were sensitive to contrast (Two-Way ANOVA, main effect contrast, each $p<0.05)$, and the relationship between contrast and neural responses was a roughly sigmoidal, monotonic-increasing function (Figure 8A). Although 17 of 36 units demonstrated some sensitivity to feedback, the effects were weak and variable and thus did not result in significant effects at the population level (Two-Way ANOVA, main effect of feedback and contrast $\times$ feedback inactivation, both $p>0.05)$.

We fit each unit's contrast sensitivity curve with the NakaRushton hyperbolic ratio function (see Materials and Methods), which explained on average over $90 \%$ of the variance across the population. We found no trends for systematic differences across feedback conditions for either the exponent parameter $n$ or the $c_{50}$ parameter (Mann-Whitney $U$-test, both $p>0.2$; Figures 8B,C). In addition, we did not detect a change in contrast response saturation across the population $\left(R_{\max }: 52 \pm 35 \mathrm{~s}^{-1}\right.$ vs. $52 \pm$ $32 \mathrm{~s}^{-1}$, feedback on vs. off respectively, $p=0.86$ ). Despite the lack of evidence for a contrast gain mechanism contributing to the effects of feedback inactivation on area summation, we tested for a correlation between changes in either $n$ or $c_{50}$ and the corresponding feedback-related changes in surround suppression ( $\Delta$ SSI from the area summation data) and found no correlation in either case: $r(\Delta$ SSI, $\Delta n)=0.03$ and $r\left(\Delta\right.$ SSI, $\left.\Delta c_{50}\right)=-0.22$ (both $p>0.1$ ). The absence of a systematic influence of feedback inactivation on contrast sensitivity within the high contrast summation field provides a second line of evidenceconsistent with the area summation analysis - that feedback does not influence area summation properties in V1 via a contrast-gain mechanism.

\section{DISCUSSION}

Our results suggest that feedback from V2 and V3 contributes to area summation properties in V1 through normalization. During feedback inactivation, we observed increased responses in V1 to large stimuli, with little or no effect on responses to small stimuli, leading to a reduction in the strength of surround suppression (Figure 3). The effects of V2/V3 cooling on the area summation curves were well explained by a normalization model in which feedback inactivation reduced the spatial extent of a divisive 
Table 4 | Comparison of area summation model and gain model.

\begin{tabular}{|c|c|c|c|c|}
\hline \multirow{3}{*}{$\begin{array}{l}\text { Free across contrast: } \\
\text { Control condition }\end{array}$} & \multirow{2}{*}{\multicolumn{2}{|c|}{$\begin{array}{l}\text { Area summation model } \\
\qquad k_{D}, k_{N}, w_{D}, w_{N}\end{array}$}} & \multirow{2}{*}{\multicolumn{2}{|c|}{$\begin{array}{c}\text { Gain model } \\
\qquad k_{D}, k_{N}\end{array}$}} \\
\hline & & & & \\
\hline & High contrast & Low contrast & High contrast & Low contrast \\
\hline Peak size $\left({ }^{\circ}\right)$ & $0.46 \pm 0.20$ & $0.71 \pm 0.65$ & $0.47 \pm 0.19$ & $0.61 \pm 0.26$ \\
\hline Peak rate $\left(s^{-1}\right)$ & $54 \pm 32$ & $32 \pm 22$ & $54 \pm 32$ & $31 \pm 21$ \\
\hline SSI (a.u.) & $0.79 \pm 0.17$ & $0.71 \pm 0.26$ & $0.78 \pm 0.19$ & $0.67 \pm 0.27$ \\
\hline$k_{D}$ (a.u.) & $1730[3110]$ & 532 [613] & $1720[3220]$ & $600[636]$ \\
\hline$k_{N}($ a.u. $)$ & 12.9 [13.8] & $5.02[5.89]$ & $13.6[16.5]$ & $4.59[5.27]$ \\
\hline$w_{D}\left({ }^{\circ}\right)$ & $0.32 \pm 0.25$ & $0.39 \pm 0.29$ & $0.34 \pm 0.17$ (fixed) & \\
\hline$w_{N}\left({ }^{\circ}\right)$ & $1.96 \pm 1.29$ & $2.14 \pm 1.31$ & $1.79 \pm 1.19$ (fixed) & \\
\hline Peak size $\left({ }^{\circ}\right)$ & $0.51 \pm 0.22$ & $0.72 \pm 0.38$ & $0.52 \pm 0.22$ & $0.65 \pm 0.32$ \\
\hline Peak rate $\left(s^{-1}\right)$ & $59 \pm 35$ & $37 \pm 27$ & $59 \pm 35$ & $36 \pm 27$ \\
\hline Asymptote size $\left({ }^{\circ}\right)$ & $4.1 \pm 1.6$ & $4.7 \pm 1.5$ & $4.1 \pm 1.5$ & $4.1 \pm 1.6$ \\
\hline Asymptote rate $\left(\mathrm{s}^{-1}\right)$ & $19 \pm 20$ & $12 \pm 14$ & $19 \pm 20$ & $14 \pm 15$ \\
\hline SSI (a.u.) & $0.71 \pm 0.22$ & $0.68 \pm 0.25$ & $0.71 \pm 0.22$ & $0.61 \pm 0.27$ \\
\hline$k_{D}$ (a.u.) & $1770[2440]$ & 450 [817] & $1830[2290]$ & 509 [857] \\
\hline$k_{N}($ a.u. $)$ & 7.99 [14.5] & 3.79 [5.82] & 10.2 [14.9] & 3.80 [4.98] \\
\hline$w_{D}\left(^{\circ}\right)$ & $0.31 \pm 0.18$ & $0.40 \pm 0.20$ & $0.36 \pm 0.22$ (fixed) & \\
\hline$w_{N}\left(^{\circ}\right)$ & $1.72 \pm 1.22$ & $2.11 \pm 1.33$ & $1.36 \pm 1.07$ (fixed) & \\
\hline Normalized $\chi^{2}$ & 4.32 & & 3.79 & \\
\hline
\end{tabular}

Mean $\pm S D$ used everywhere except for $k_{D}$ and $k_{N}$ reported as median [IQR]. Some results from Table 1 are repeated here for convenience.

normalization pool (Figures 2, 3). Contrast-dependent effects on area summation were unaltered during feedback inactivation (Figure 6), as was contrast sensitivity within the receptive field center (Figure 8), providing evidence that feedback acts independently of previously described contrast-gain mechanisms known to exist in V1 (Sclar et al., 1990; Levitt and Lund, 1997; Cavanaugh et al., 2002).

The original normalization model (Heeger, 1992) was put forward to account for two shortcomings of the Hubel-Wiesel linear model of simple cells (Hubel and Wiesel, 1962): (1) response saturation at high contrast and (2) non-specific suppression of responses due to additional stimuli in or near the classical receptive field. Although the normalization model accounts for both processes with the same equation, recent evidence indicates that the two may in fact be mechanistically distinct (Olsen et al., 2010): Working in the olfactory system of Drosophila, these investigators demonstrated that response saturation occurred within each glomerular channel, while normalization was due to lateral inhibitory interactions between glomeruli. Our results show a similar dissociation. We found that feedback inactivation reduced surround suppression solely by shrinking the spatial extent of the normalization pool and, remarkably, that this effect was independent of the gain modulations that accounted for effects of stimulus contrast on area summation. We further confirmed that contrast sensitivity within the classical receptive field is unaltered when inactivating feedback. Insofar as divisive normalization underlies area summation, our results provide direct evidence that corticocortical feedback participates in normalization independent of the circuit mechanisms involved in contrast-gain control. It is nevertheless important to note that we applied normalization as a premise for interpreting these data. Therefore, our results do not rule out alternative models that account for summation properties (e.g., Sceniak et al., 1999).

Based on our data, feedback from V2 and V3 interacts preferentially with the pool of neurons that produces surround suppression in $\mathrm{V} 1$ rather than the pool that provides the excitatory drive. This is consistent with earlier propositions that feedback acts as a modulator of neural activity as opposed to a driver (Shao and Burkhalter, 1996; Sherman and Guillery, 1998; Angelucci and Bressloff, 2006). Such a characterization is based largely on anatomical work which has shown that feedback axons tend to target the distal apical dendrites of pyramidal neurons (Rockland and Virga, 1989; Anderson and Martin, 2009) and are therefore capable of having an impact on the gain of neuronal outputs (Larkum et al., 2004). However, here we have provided evidence contrary to the idea that feedback modulates gain, but rather that it sets the size of the suppressive field. The idea that feedback's role as a modulator can be suppressive has not been fully appreciated 

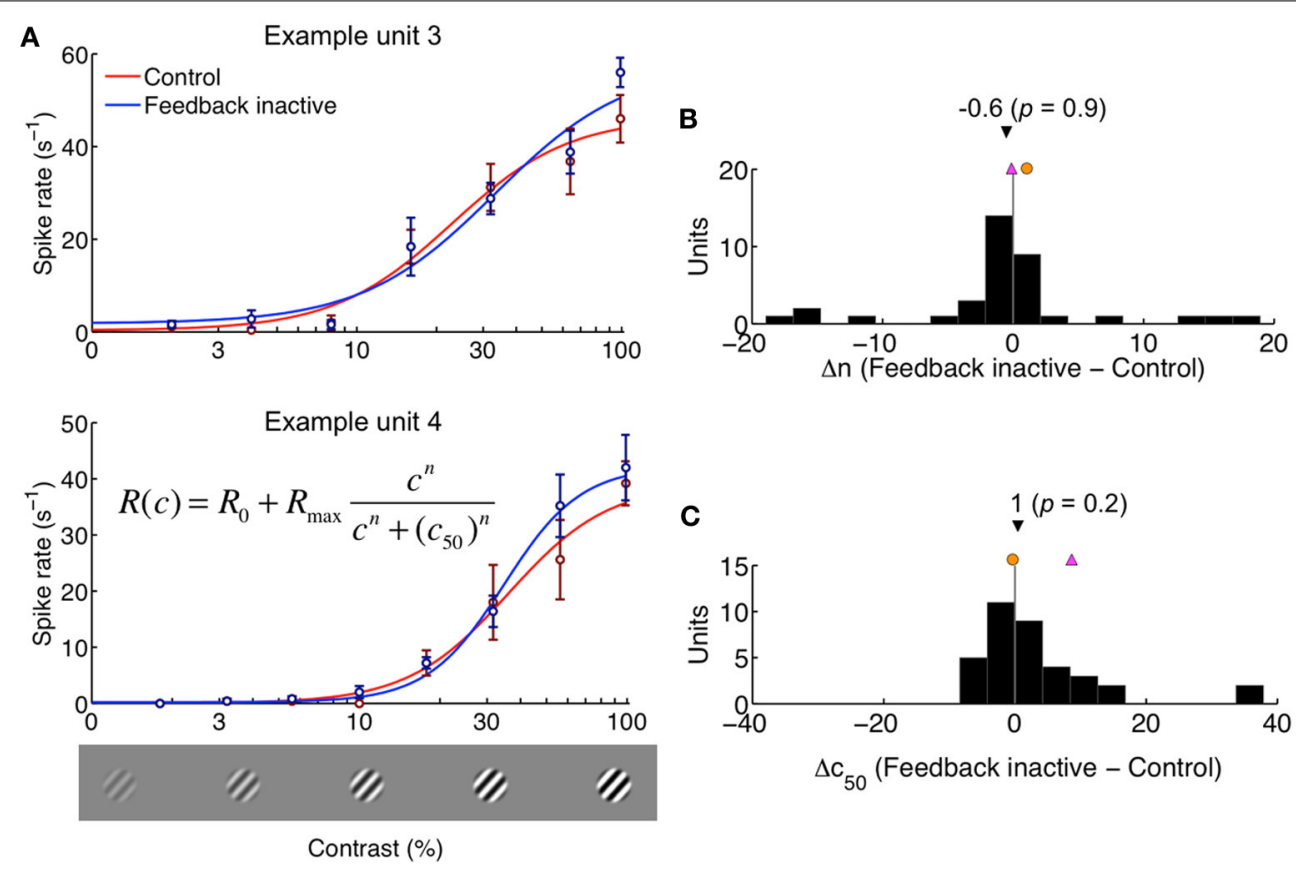

FIGURE 8 | Contrast sensitivity is unaltered by feedback inactivation. (A) Contrast response functions plotted for two example units. The grating was restricted to the center of the receptive field. Data are shown as mean \pm s.e.m., and curves were obtained using the Naka-Rushton model (inset equation) by fitting the parameters $n$ and $c_{50}$. The spike rates for spontaneous activity $\left(R_{0}\right)$ and maximum response $\left(R_{\max }\right)$ were measured.
(B) Difference in parameter $n$ between Feedback inactive and Control conditions. (C) Difference in parameter $C_{50}$ between Feedback inactive and Control conditions. Black triangle indicates population median (with two-tailed Mann-Whitney $U$-test $p$-value). Magenta and orange symbols indicate shifts for example units shown in (A). Feedback-inactivation did not lead to a systematic effect in contrast response function parameters. until recently (Nassi et al., 2013). Previously, one of the only clues regarding feedback's involvement in area summation was the relatively fast response facilitation from far regions of the receptive field surround (Ichida et al., 2007). Surround suppression could be mediated by feedback connections directly onto local inhibitory neurons (Gonchar and Burkhalter, 2003; Anderson and Martin, 2009). This would be consistent with recent work in the rodent, showing that somatostatin-positive inhibitory neurons play an important role in surround suppression (Adesnik et al., 2012) and that long range excitatory projections can have a net suppressive effect through the recruitment of local inhibitory neurons (Iurilli et al., 2012; Palmer et al., 2012). Suppression through feedback could also be mediated indirectly via horizontally projecting excitatory neurons in superficial layers which are known to target inhibitory neurons (McGuire et al., 1991) and have been implicated in several forms of "top-down" modulatory influences (Ito and Gilbert, 1999; Li et al., 2004; Ramalingam et al., 2013). Feedback need not rely solely on inhibitory neurons in order to produce suppression, as increases in conductance as well as synaptic depression can lead to reduced neuronal output without a net increase in inhibition. In fact, recent evidence suggests that surround suppression relies on the dynamic interplay between highly interconnected excitatory and inhibitory networks, such as an inhibition-stabilized network, and there are likely to be many ways by which feedback could modulate such a balanced network regime (Ozeki et al., 2009; Haider et al., 2010).
In distinction to inputs from the normalization pool, driving inputs received by V1 neurons are thought to establish basic selectivity for such properties as spatial position and orientation, and are likely mediated primarily by feed-forward connections (Priebe and Ferster, 2012). In support of this idea is our previously reported observation that orientation preference within the receptive field center is unaffected during feedback inactivation (Nassi et al., 2013). However, we did find that selectivity for orientation was often reduced due to slightly increased responses to orthogonal orientations when feedback was inactivated. This is consistent with models of orientation tuning that depend on local cortical circuits to sharpen orientation preference (Ben-Yishai et al., 1995; Somers et al., 1995; Troyer et al., 1998); an operation that may also rely on normalization (Heeger, 1992).

According to our modeling results, feedback from V2 and V3 contributes to the overall size but not the gain of normalization in V1. Specifically, feedback increases the spatial extent over which normalization operates. An increase in the size of the normalization pool leads to increased suppression in the surround while leaving responses within the receptive field center unchanged (Figure 2F). This is consistent with both the area summation data and center contrast response function data presented here (Figures 1, 8), as well as the fact that receptive fields increase in size along the visual cortical hierarchy. Feedback from V2 and V3 can cover aggregate visual fields five-ten times the size of the receptive field center in V1 (Angelucci and Bressloff, 2006). This may explain why observed effects of feedback inactivation were 
strongest for stimulus diameters approximately two-eight times the size of the receptive field center and relatively weaker for the largest stimulus diameters tested (Nassi et al., 2013), which likely evoked stronger responses from areas such as MT that remained active during cooling of V2/V3 (Ponce et al., 2008). Indeed, substantial suppression remained during feedback inactivation, not only for the largest stimulus diameters tested, but also for stimulus diameters that extended just beyond the center of the receptive field. These results suggest that, in addition to feedback from $\mathrm{V} 2 / \mathrm{V} 3$, other cortical sources of feedback, as well as horizontal connections intrinsic to $\mathrm{V} 1$, all combine to produce the level of surround suppression observed under normal conditions. Each of these distinct sources of suppression likely operates at different spatio-temporal scales (Bair et al., 2003; Angelucci and Bressloff, 2006; Reynaud et al., 2012) and their interaction with one another within the context of normalization will be an important question to be addressed in the course of future research.

Area summation properties are not only dependent on topdown feedback signals but also on bottom-up stimulus properties such as luminance contrast (Levitt and Lund, 1997). As stimulus contrast is reduced, the size of the summation field increases. This was first accounted for with a difference of Gaussians model in which a reduction in contrast increases the spatial extent of excitatory drive (Sceniak et al., 1999). In subsequent work, it was shown that the increase in summation field size at low contrast could be more parsimoniously explained by a divisive normalization model in which input drive and the normalization pool are stable in spatial extent and only their relative gains depend on contrast (Cavanaugh et al., 2002). Our analysis reproduced the contrast gain results of the Cavanaugh model (Figure 7). The current results further showed that inactivation of feedback leaves contrast-gain effects on area summation unaltered (Figure 6). This suggests that the effects of contrast on area summation are mediated by circuit mechanisms independent of feedback. One likely candidate would be horizontal connections intrinsic to $\mathrm{V} 1$, which have been shown to cover the necessary visuotopic extent (Angelucci and Bressloff, 2006). Indeed, recent evidence from voltage-sensitive dye imaging in alert macaques suggests that horizontal connections may act to dynamically clamp local contrast gain mechanisms (Reynaud et al., 2012). Interestingly, these investigators were able to account for their observed results with two distinct normalization processes, proposing that recurrent polysynaptic intracortical loops mediate contrast gain and long-range monosynaptic horizontal spread mediates surround suppression. Taken together, our results suggest that horizontal connections in V1 may set the gain of normalization in V1, whereas feedback sets the extent over which horizontal connections are effective-essentially gating horizontal signals depending on stimulus conditions and behavioral state (Gilbert and Sigman, 2007). This view is consistent with the idea that feedback can modify the "association field" that is likely comprised of local horizontal inputs (Field et al., 1993; Ramalingam et al., 2013).

Recent studies on attention have provided increasing support for the idea that feedback modulates properties of spatial integration (Roberts et al., 2007; Anton-Erxleben et al., 2009; Sundberg et al., 2009). For example, in V4 attention directed to the receptive field center weakened suppression from the surround, while attention to the surround enhanced suppression
(Sundberg et al., 2009). These effects were accounted for by a normalization model similar to the one considered here (Lee and Maunsell, 2009; Reynolds and Heeger, 2009). In V1, effects of attention have been found to be more complicated: some studies have found that attention reduces the impact of the surround, whereas others have found that the impact of the surround is enhanced (Ito and Gilbert, 1999; Roberts et al., 2007; Chen et al., 2008; Poort et al., 2012). These effects appear to depend on several factors including stimulus eccentricity, whether surround stimuli are facilitatory or suppressive and the nature of the perceptual task. In the current study, the behavioral task was simply to fixate a central cross, leaving the attentional focus of the animals uncontrolled and thus indeterminate. It is probable, however, that the receptive fields we studied fell outside of the locus of attention which was most likely directed toward the fixation point-a configuration for which suppressive effects are expected. A prediction for future studies is that training the animal to direct attention toward the receptive fields under study might uncover excitatory influences of feedback.

\section{ACKNOWLEDGMENTS}

This work was supported by National Institutes of Health EY11379, EY12196, and the National Science Foundation (NSF08-557). We thank Alexandra Smith for excellent technical assistance; John A. Assad and Rachel I. Wilson for helpful comments on this manuscript. Use of the Orchestra High Performance Compute Cluster at Harvard Medical School was partially funded through grant NCRR 1S10RR028832-01.

\section{SUPPLEMENTARY MATERIAL}

The Supplementary Material for this article can be found online at: http://www.frontiersin.org/journal/10.3389/fnsys.2014. 00105/abstract

\section{REFERENCES}

Adesnik, H., Bruns, W., Taniguchi, H., Huang, Z. J., and Scanziani, M. (2012). A neural circuit for spatial summation in visual cortex. Nature 490, 226-231. doi: 10.1038 /nature11526

Albright, T. D., and Stoner, G. R. (2002) Contextual influences on visual processing. Annu. Rev. Neurosci. 25, 339-379. doi: 10.1146/annurev.neuro.25.112701.142900

Anderson, J. C., and Martin, K. A. (2009). The synaptic connections between cortical areas V1 and V2 in macaque monkey. J. Neurosci. 29, 11283-11293. doi: 10.1523/JNEUROSCI.5757-08.2009

Angelucci, A., and Bressloff, P. C. (2006). Contribution of feedforward, lateral and feedback connections to the classical receptive field center and extra-classical receptive field surround of primate V1 neurons. Prog. Brain Res. 154, 93-120. doi: 10.1016/S0079-6123(06)54005-1

Anton-Erxleben, K., Stephan, V. M., and Treue, S. (2009). Attention reshapes center-surround receptive field structure in macaque cortical area MT. Cereb. Cortex 19, 2466-2478. doi: 10.1093/cercor/bhp002

Bair, W., Cavanaugh, J. R., and Movshon, J. A. (2003). Time course and timedistance relationship for surround suppression in macaque V1 neurons. J. Neurosci. 23, 7690-7701.

Ben-Yishai, R., Bar-Or, R. L., and Sompolinsky, H. (1995). Theory of orientation tuning in visual cortex. Proc. Natl. Acad. Sci. U.S.A. 92, 3844-3848. doi: 10.1073/pnas.92.9.3844

Carandini, M., and Heeger, D. J. (2012). Normalization as a canonical neural computation. Nat. Rev. Neurosci. 13, 51-62. doi: 10.1038/nrn3136

Carandini, M., Heeger, D. J., and Movshon, J. A. (1997). Linearity and normalization in simple cells of the macaque primary visual cortex. J. Neurosci. 17, $8621-8644$ 
Cavanaugh, J. R., Bair, W., and Movshon, J. A. (2002). Nature and interaction of signals from the receptive field center and surround in macaque V1 neurons. J. Neurophysiol. 88, 2530-2546. doi: 10.1152/jn.00692.2001

Cavanaugh, J. R., Joiner, W. M., and Wurtz, R. H. (2012). Suppressive surrounds of receptive fields in monkey frontal eye field. J. Neurosci. 32, 12284-12293. doi: 10.1523/JNEUROSCI.0864-12.2012

Chen, Y., Martinez-Conde, S., Macknik, S. L., Bereshpolova, Y., Swadlow, H. Z., and Alonso, J. M. (2008). Task difficulty modulates the activity of specific neuronal populations in primary visual cortex. Nat. Neurosci. 11, 974-982. doi: $10.1038 / \mathrm{nn} .2147$

Field, D. J., Hayes, A., and Hess, R. F. (1993). Contour integration by the human visual system: evidence for a local “association field." Vis. Res. 33, 173-193. doi: 10.1016/0042-6989(93)90156-Q

Gilbert, C. D., and Sigman, M. (2007). Brain states: top-down influences in sensory processing. Neuron 54, 677-696. doi: 10.1016/j.neuron.2007.05.019

Gonchar, Y., and Burkhalter, A. (2003). Distinct GABAergic targets of feedforward and feedback connections between lower and higher areas of rat visual cortex. J. Neurosci. 23, 10904-10912.

Haider, B., Krause, M. R., Duque, A., Yu, Y., Touryan, J., Mazer, J. A., et al. (2010). Synaptic and network mechanisms of sparse and reliable visual cortical activity during nonclassical receptive field stimulation. Neuron 65, 107-121. doi: 10.1016/j.neuron.2009.12.005

Heeger, D. J. (1992). Normalization of cell responses in cat striate cortex. Vis. Neurosci. 9, 181-197. doi: 10.1017/S0952523800009640

Hoel, P. G., Port, S. C., and Stone, C. J. (1971). Introduction to Statistical Theory. Boston: Houghton-Mifflin.

Hubel, D. H., and Wiesel, T. N. (1962). Receptive fields, binocular interaction and functional architecture in the cat's visual cortex. J. Physiol. 160, 106-154.

Ichida, J. M., Schwabe, L., Bressloff, P. C., and Angelucci, A. (2007). Response facilitation from the "suppressive" receptive field surround of macaque V1 neurons. J. Neurophysiol. 98, 2168-2181. doi: 10.1152/jn.00298.2007

Ito, M., and Gilbert, C. D. (1999). Attention modulates contextual influences in the primary visual cortex of alert monkeys. Neuron 22, 593-604. doi: 10.1016/S0896-6273(00)80713-8

Iurilli, G., Ghezzi, D., Olcese, U., Lassi, G., Nazzaro, C., Tonini, R., et al. (2012). Sound-driven synaptic inhibition in primary visual cortex. Neuron 73, 814-828. doi: 10.1016/j.neuron.2011.12.026

Kayser, A., Priebe, N. J., and Miller, K. D. (2001). Contrast-dependent nonlinearities arise locally in a model of contrast-invariant orientation tuning. J. Neurophysiol. 85:2130-2149.

Larkum, M. E., Senn, W., and Luscher, H. R. (2004). Top-down dendritic input increases the gain of layer 5 pyramidal neurons. Cereb. Cortex 14, 1059-1070. doi: 10.1093/cercor/bhh065

Lee, J., and Maunsell, J. H. (2009). A normalization model of attentional modulation of single unit responses. PLOS ONE 4:e4651. doi: 10.1371/journal.pone.0004651

Levitt, J. B., and Lund, J. S. (1997). Contrast dependence of contextual effects in primate visual cortex. Nature 387, 73-76. doi: 10.1038/387073a0

Li, W., Piech, V., and Gilbert, C. D. (2004). Perceptual learning and top-down influences in primary visual cortex. Nat. Neurosci. 7, 651-657. doi: 10.1038/nn1255

Lomber, S. G., Payne, B. R., and Horel, J. A. (1999). The cryoloop: an adaptable reversible cooling deactivation method for behavioral or electrophysiological assessment of neural function. J. Neurosci. Methods 86, 179-194. doi: 10.1016/S0165-0270(98)00165-4

McGuire, B. A., Gilbert, C. D., Rivlin, P. K., and Wiesel, T. N. (1991). Targets of horizontal connections in macaque primary visual cortex. J. Comp. Neurol. 305, 370-392. doi: 10.1002/cne.903050303

Naka, K. I., and Rushton, W. A. H. (1966). S-potentials from luminosity units in the retina of fish (cyprinidae). J. Physiol. 185, 587-599.

Nassi, J. J., Lomber, S. G., and Born, R. T. (2013). Corticocortical feedback contributes to surround suppression in V1 of the alert primate. J. Neurosci. 33, 8504-8517. doi: 10.1523/JNEUROSCI.5124-12.2013

Nienborg, H., Hasenstaub, A., Nauhaus, I., Taniguchi, H., Huang, Z. J., and Callaway, E. M. (2013). Contrast dependence and differential contributions from somatostatin- and parvalbumin-expressing neurons to spatial integration in mouse V1. J. Neurosci. 33, 11145-11154. doi: 10.1523/JNEUROSCI.532012.2013

Olsen, S. R., Bhandawat, V., and Wilson, R. I. (2010). Divisive normalization in olfactory population codes. Neuron 66, 287-299. doi: 10.1016/j.neuron.2010.04.009
Ozeki, H., Finn, I. M., Schaffer, E. S., Miller, K. D., and Ferster, D. (2009). Inhibitory stabilization of the cortical network underlies visual surround suppression. Neuron 62, 578-592. doi: 10.1016/j.neuron.2009.03.028

Palmer, L. M., Schulz, J. M., Murphy, S. C., Ledergerber, D., Murayama, M., and Larkum, M. E. (2012). The cellular basis of $\mathrm{GABA}_{B}$-mediated interhemispheric inhibition. Science 335, 989-993. doi: 10.1126/science.1217276

Ponce, C. R., Lomber, S. G., and Born, R. T. (2008). Integrating motion and depth via parallel pathways. Nat. Neurosci. 11, 216-223. doi: 10.1038/nn2039

Poort, J., Raudies, F., Wannig, A., Lamme, V. A. F., Neumann, H., and Roelfsema, P. (2012). The role of attention in figure-ground segregation in areas V1 and V4 of the visual cortex. Neuron 75, 143-156. doi: 10.1016/j.neuron.2012. 04.032

Priebe, N. J., and Ferster, D. (2012). Mechanisms of neuronal computation in mammalian visual cortex. Neuron 75, 194-208. doi: 10.1016/j.neuron.2012. 06.011

Ramalingam, N., McManus, J. N., Li, W., and Gilbert, C. D. (2013). Top-down modulation of lateral interactions in visual cortex. J. Neurosci. 33, 1773-1789. doi: 10.1523/JNEUROSCI.3825-12.2013

Reynaud, A., Masson, G. S., and Chavane, F. (2012). Dynamics of local input normalization result from balanced short- and long-range intracortical interactions in area V1. J. Neurosci. 32, 12558-12569. doi: 10.1523/JNEUROSCI.161812.2012

Reynolds, J. H., and Heeger, D. J. (2009). The normalization model of attention. Neuron 61, 168-185. doi: 10.1016/j.neuron.2009.01.002

Roberts, M., Delicato, L. S., Herrero, J., Gieselmann, M. A., and Thiele, A. (2007). Attention alters spatial integration in macaque V1 in an eccentricity-dependent manner. Nat. Neurosci. 10, 1483-1491. doi: 10.1038/nn1967

Rockland, K. S., and Virga, A. (1989). Terminal arbors of individual "feedback" axons projecting from area $\mathrm{V} 2$ to $\mathrm{V} 1$ in the macaque monkey: a study using immunohistochemistry of anterogradely transported Phaseolus vulgaris-leucoagglutinin. J. Comp. Neurol. 285, 54-72. doi: 10.1002/cne. 902850106

Sceniak, M. P., Ringach, D. L., Hawken, M. J., and Shapley, R. (1999). Contrast's effect on spatial summation by macaque V1 neurons. Nat. Neurosci. 2, 733-739. doi: $10.1038 / 11197$

Sclar, G., Maunsell, J. H., and Lennie, P. (1990). Coding of image contrast in central visual pathways of the macaque monkey. Vision Res. 30, 1-10. doi: 10.1016/0042-6989(90)90123-3

Shao, Z., and Burkhalter, A. (1996). Different balance of excitation and inhibition in forward and feedback circuits of rat visual cortex. J. Neurosci. 16, 7353-7365.

Sherman, S. M., and Guillery, R. W. (1998). On the actions that one nerve cell can have on another: distinguishing "drivers" from "modulators." Proc. Natl. Acad. Sci. U.S.A. 95, 7121-7126. doi: 10.1073/pnas.95.12.7121

Somers, D. C., Nelson, S. B., and Sur, M. (1995). An emergent model of orientation selectivity in cat visual cortical simple cells. J. Neurosci. 15, 5448-5465.

Sundberg, K. A., Mitchell, J. F., and Reynolds, J. H. (2009). Spatial attention modulates center-surround interactions in macaque visual area V4. Neuron 61, 952-963. doi: 10.1016/j.neuron.2009.02.023

Troyer, T. W., Krukowski, A. E., Priebe, J. N., and Miller, K. D. (1998). Contrastinvariant orientation tuning in cat visual cortex: thalamocortical input tuning and correlation-based intracortical connectivity. J. Neurosci. 18, 5908-5927.

Vaiceliunaite, A., Eriksen, S., Franzen, F., Katzner, S., and Busse, L. (2013). Spatial integration in mouse primary visual cortex. J. Neurophysiol. 110, 964-972. doi: 10.1152/jn.00138.2013

Conflict of Interest Statement: The authors declare that the research was conducted in the absence of any commercial or financial relationships that could be construed as a potential conflict of interest.

Received: 12 March 2014; accepted: 13 May 2014; published online: 30 May 2014. Citation: Nassi JJ, Gómez-Laberge C, Kreiman G and Born RT (2014) Corticocortical feedback increases the spatial extent of normalization. Front. Syst. Neurosci. 8:105. doi: 10.3389/fnsys.2014.00105

This article was submitted to the journal Frontiers in Systems Neuroscience. Copyright (c) 2014 Nassi, Gómez-Laberge, Kreiman and Born. This is an openaccess article distributed under the terms of the Creative Commons Attribution License (CC BY). The use, distribution or reproduction in other forums is permitted, provided the original author(s) or licensor are credited and that the original publication in this journal is cited, in accordance with accepted academic practice. No use, distribution or reproduction is permitted which does not comply with these terms. 